\title{
Particle-modified electrodes: General mass transport theory, experimental validation, and the role of electrostatics
}

\author{
Lifu Chen, Enno Kätelhön, and Richard G. Compton* \\ Department of Chemistry, Physical and Theoretical Chemistry Laboratory, Oxford University, South Parks
} Road, Oxford, OX1 3QZ, United Kingdom

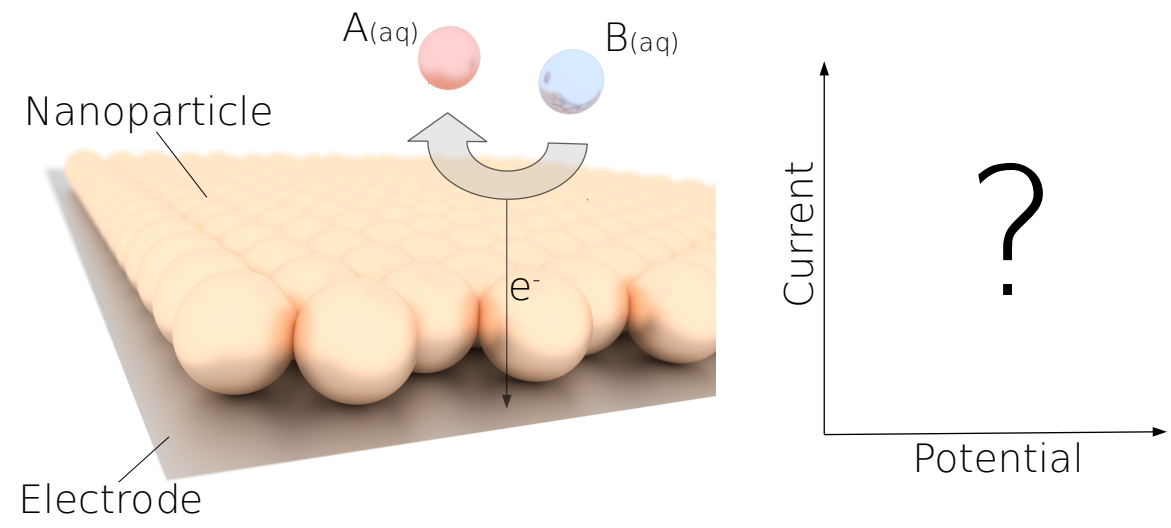

\begin{abstract}
We present general mass transport theory for cyclic voltammetry at electrodes that are partially 'blocked' with electrochemically-inactive particles. It is broadly applicable covering reversible, quasireversible, and irreversible reactions and is experimentally validated using the hydrazine oxidation where theory follows experiment in quantitative detail. We extend our analysis to explore the role of electrostatics in three further reactions where the voltammetry is startlingly altered by electrostatic effects: the reduction of hexaammineruthenium (III), the oxidation of ferrocenemethanol, and the oxidation of ferrocyanide.
\end{abstract}

*Corresponding author: Richard G. Compton, richard.compton@chem.ox.ac.uk 


\section{Introduction}

Electrodes modified with micro- or nanoparticles bear enormous potential in the development of new materials in electrocatalysis and in sensing and are hence subject to extensive research activities. Progress and opportunities in the research on nanostructured electrodes are regularly reviewed and we refer the interested reader to related literature [1-6].

Recent work $[7,8]$ has started to qualitatively demonstrate the complexity of any voltammetric analysis at particle-modified electrodes. Depending on the electrode geometry and the time scale at which the electrode potential is altered, the presence of the particles may induce voltammetric thin-layer effects or retard the mass transport towards the electrode. In cyclic voltammetry this may lead to altered peak currents and shifted peak potentials which may falsely be interpreted as positive or negative catalysis: Earlier "peak onsets" and greater peak currents may suggest the modifying particles were catalytically active despite the observations being solely due to changed mass transport characteristics. On the contrary, one might infer negative catalysis from a "later" occurrence of the peak at greater overpotentials despite a possible positive catalytic activity of the particles where 'catalysis' implies a change of a rate constant. In either case, complementary theoretical modelling is essential in the analysis of the voltammetric data.

While our prior studies of particle-modified electrodes have discussed reversible reactions to ensure the the analysis focusses exclusively on mass transport effects, we here present a comprehensive analysis of particle modified electrodes considering both partial 'blocking' effects and electrostatic interactions between charged reactants and products and the blocking particles, assuming Butler Volmer kinetics.

Theoretical results are first introduced and reveal the complex dependency of the peak current and the peak potential on the scan rate and the number of particles deposited. Our theory is then validated via experimental data of the hydrazine oxidation which shows excellent agreement between theory and experiment. Finally, we turn to three other reactions (hexaammineruthenium (III) reduction, ferrocenemethanol oxidation, and ferrocyanide oxidation) which differ from hydrazine in that there is an electrostatic interaction between the species involved in the reaction and the surface of the deposited particles. A startlingly wide range of voltammograms is seen.

\section{Experimental}

The following section first details the chemicals used in the experimental work. We then report the procedure followed in the synthesis of the Nafion particles and finally explain the electrochemical measurements.

\subsection{Chemicals}

All chemical reagents were used as received without further purification: hydrazine monohydrate $\left(\mathrm{N}_{2} \mathrm{H}_{4} \cdot \mathrm{H}_{2} \mathrm{O}, 98 \%\right.$, Sigma-Aldrich), hexaammineruthenium(III) chloride $\left(\left[\mathrm{Ru}\left(\mathrm{NH}_{3}\right)_{6}\right] \mathrm{Cl}_{3}, \mathrm{Ru} \geq 32.1\right.$ $\%$, Alfa Aesar), potassium ferrocyanide trihydrate $\left(\mathrm{K}_{4}\left[\mathrm{Fe}(\mathrm{CN})_{6}\right] 3 \mathrm{H}_{2} \mathrm{O}, \geq 99 \%\right.$, AnalaR NORMAPUR), ferrocenemethanol ( $\mathrm{FcCH}_{2} \mathrm{OH}, 97 \%$, Santa Cruz Biotechnology), potassium chloride ( $\mathrm{KCl}, \geq$ $99.0 \%$, Sigma-Aldrich), sodium chloride ( $\mathrm{NaCl}, \geq 99.5 \%$, Sigma-Aldrich), sodium phosphate dibasic $\left(\mathrm{Na}_{2} \mathrm{HPO}_{4}, \geq 99.0 \%\right.$, Sigma-Aldrich $)$ and potassium phosphate monobasic $\left(\mathrm{KH}_{2} \mathrm{PO}_{4}, \geq 99.0 \%\right.$, Sigma-Aldrich). Nafion perfluorinated resin solution (5 wt\% in lower aliphatic alcohol and water with a water content of $45 \%$, Sigma-Aldrich) was bathed in water at $52 \pm 0.5^{\circ} \mathrm{C}$ to obtain a concentrated 12.5 wt\% Nafion solution. Phosphate buffered saline solution (PBS, $\mathrm{pH}=7.4$ ) was composed of 137 
$\mathrm{mM} \mathrm{NaCl}, 2.7 \mathrm{mM} \mathrm{KCl}, 10 \mathrm{mM} \mathrm{Na}_{2} \mathrm{HPO}_{4}$ and $1.8 \mathrm{mM} \mathrm{KH}_{2} \mathrm{PO}_{4}$. All solutions were prepared using ultrapure water (Millipore) with a resistivity of $18.2 \Omega \mathrm{cm}$ at $298 \mathrm{~K}$ and degassed with Argon before use.

\subsection{Synthesis of Nafion particles}

Nafion particles [8-11] were synthesized by the re-precipitation method [9] as described previously. Briefly, $50 \mu \mathrm{L}$ concentrated $12.5 \mathrm{wt} \%$ Nafion was injected dropwise into $1 \mathrm{~mL}$ ultrapure water under stirring by magnetic vortexing at $600 \mathrm{rpm}$ for 5 minutes. The resulting mixture was subjected to sonication (FB15050, Fisher Scientific, 50/60 Hz, Germany) for $30 \mathrm{~min}$ at room temperature. The suspension was then centrifuged (Eppendorf Centrifuge $5430 \mathrm{R}$ ) at $14000 \mathrm{rpm}$ for $10 \mathrm{~min}$ and the precipitate was dispersed evenly in $0.5 \mathrm{~mL}$ water by sonication for $5 \mathrm{~min}$. A stable suspension of sub-micron sized Nafion particles with an average radius of $0.43 \pm 0.26 \mu \mathrm{m}$ [9] was obtained.

\subsection{Electrochemical measurements}

Electrochemical experiments were performed with an Autolab II potentiostat (Metrohm-Autolab BV, Netherlands) and a three-electrode set-up inside a grounded and thermostatted $(25.0 \pm 0.5$ ${ }^{\circ} \mathrm{C}$ ) Faraday cage. A glassy carbon macroelectrode (GC, $3 \mathrm{~mm}$ diameter) was used as the working electrode, a saturated calomel electrode (SCE) as the reference electrode and a graphite rod as the counter electrode. Prior to each voltammetric scan, the working electrode was polished using micropolish alumina (Buehler) of decreasing particle size $(1.0,0.3$ and $0.05 \mu \mathrm{m})$ followed by sonication in water and drying with nitrogen. $10 \mu \mathrm{L}$ of varying concentrations of fresh prepared Nafion particles suspension was drop-casted on the GC macroelectrode and left to dry under a nitrogen environment. Cyclic voltammetry was conducted at selected scan rates of between $25 \mathrm{mV} \mathrm{s}^{-1}$ to $800 \mathrm{mV} \mathrm{s}^{-1}$ in $1.0 \mathrm{mM}$ hydrazine support with PBS buffer, $1.0 \mathrm{mM}$ hexaammineruthenium(III) chloride supported with $0.1 \mathrm{M} \mathrm{KCl}$, ferrocenemethanol supported with $0.1 \mathrm{M} \mathrm{KCl}$, and $1.0 \mathrm{mM}$ potassium ferrocyanide supported with $0.1 \mathrm{M} \mathrm{KCl}$.

\section{Computational}

This section commences with the introduction of our theoretical model and the computational methods that are used to solve the resulting partial differential equations. We then introduce the dimensionless coordinates that are employed in the presentation of some of the theoretical findings and facilitate an easier comparison with experimental data. The section concludes with the details and the specifics of the theoretical model when tailored to the hydrazine oxidation reaction.

\subsection{Theoretical model}

We model cyclic voltammetry at a macroelectrode. The species $B$ is herein oxidised to the species $A$,

$$
B-e \rightarrow A
$$

at a bare electrode and an electrode decorated with a variable number of layers of spheres. These layers are complete and comprise identical spheres that are arranged in a hexagonal packing (see Figure 1a). The spheres are solid, electrochemically inactive, and neither electroactive species nor electrolyte- nor solvent molecules can adsorb on their surfaces. 
a)

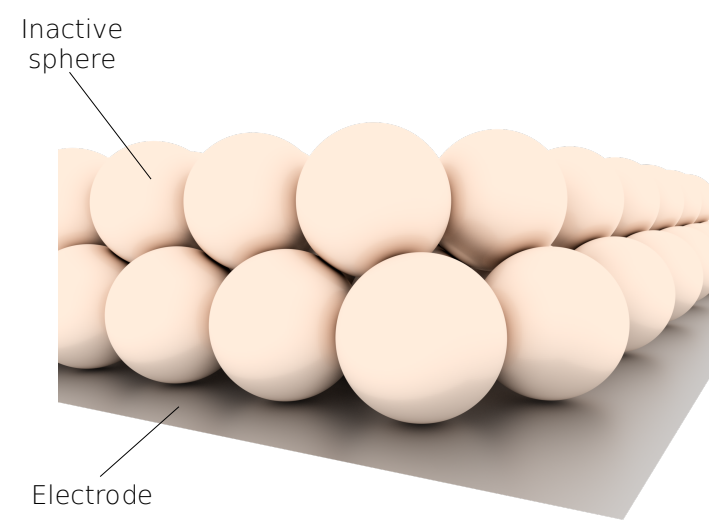

b)

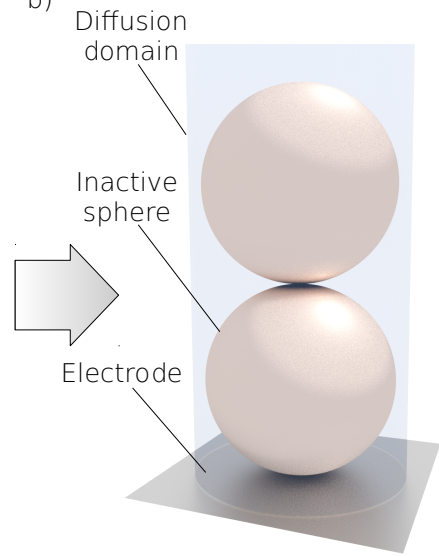

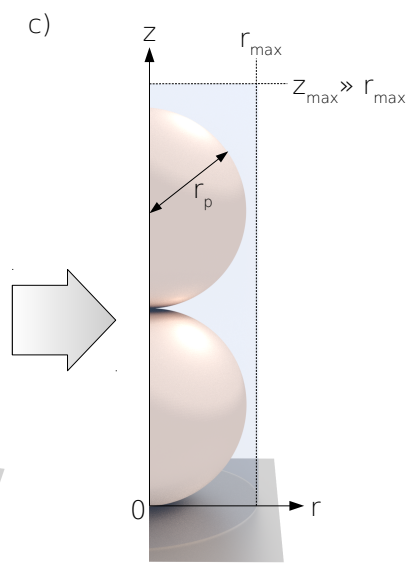

Figure 1: Illustration of the diffusion domain approximation as applied to the hexagonal packing of spheres. The figure is reproduced from our previous study [8].

The theoretical model is widely identical with the theoretical model we used in our previous studies $[7,8]$ where it has been described exhaustively and the sole difference lies in the electrode kinetics. While the previous work exclusively discussed reversible reactions, the reaction kinetics here are modelled as a quasi-reversible single-electron reaction and described by the Butler-Volmer equation.

In short, the geometry depicted in Figure 1a is simplified along the lines of the diffusion domain approximation [12] which reduces the dimensionality of the modelled space from three to two (see Figure $1 \mathrm{~b}$ and $1 \mathrm{c}$ ). The height $z_{\max }$ of the simulated space is chosen to be sufficiently large for reactions at the electrode not to affect concentrations at $z_{\max }$. The radius $r_{\max }$ of the diffusion domain is set to 1.05 times the radius of the particles which is a value that has priorly [8] been established on the basis of theoretical considerations. Mass transport is exclusively due to diffusion and described by Fick's second law:

$$
\frac{\partial c_{A}(r, z, t)}{\partial t}=D \Delta c_{A}(r, z, t) \text { and } \frac{\partial c_{B}(r, z, t)}{\partial t}=D \Delta c_{B}(r, z, t)
$$

where $c_{A}$ and $c_{B}$ are the concentrations of the species $A$ and $B$, respectively, and $D$ is the diffusion coefficient shared by both species. Throughout the entire simulation, the concentrations of species A and $\mathrm{B}$ in the bulk solution remain constant:

$$
\begin{aligned}
& c_{A}(z \rightarrow \infty)=0 \\
& c_{B}(z \rightarrow \infty)=c^{*}
\end{aligned}
$$

The electrode kinetics follow Butler-Volmer kinetics for a one-electron transfer process:

$$
\begin{aligned}
\frac{I(t)}{F} & =-D \int_{S} d A \frac{\partial c_{A}(r, z=0, t)}{\partial t} \\
& =D \int_{S} d A \frac{\partial c_{B}(r, z=0, t)}{\partial t} \\
& =-\int_{S} d A k_{0}\left(e^{-\alpha_{c} \theta} c_{A}(r, z=0, t)-e^{\alpha_{a} \theta} c_{B}(r, z=0, t)\right)
\end{aligned}
$$




\begin{tabular}{c|c|c} 
Parameter & Symbol (dimensioned) & Symbol (dimensionless) and transformation \\
\hline Radial coordinate & $r$ & $R=\frac{r}{r_{p}}$ \\
Axial coordinate & $z$ & $Z=\frac{z}{r_{p}}$ \\
Time & $t$ & $T=\frac{D}{r_{p}^{2}} t$ \\
Potential & $E$ & $\theta=\frac{F}{R T}\left(E-E_{f}^{0}\right)$ \\
Scan rate & $v$ & $\sigma=\frac{r_{p}^{2} F}{D R T} v$ \\
Electrochemical rate constant & $k_{0}$ & $K_{0}=\frac{r_{p}}{D} k_{0}$ \\
Concentration & $c$ & $C=\frac{c}{c^{*}}$ \\
Current & $I$ & $J=\left(\frac{r_{\max }}{r_{e l}}\right)^{2} \frac{I}{F D r_{p} c^{*}}=\int_{S} d A\left(-\frac{\partial C}{\partial Z}\right)$
\end{tabular}

Table 1: Dimensionless coordinates used in this study. $r_{p}$ is the particle radius, $c^{*}$ is the bulk concentration of the initially present electroactive species $\mathrm{B}, r_{e}$ is the radius of the macroelectrode, and $S$ is the surface of the electrode in the diffusion domain approximation.

with:

$$
\theta=\frac{F}{R T}\left(E-E_{f}^{0}\right) \text { and } \quad \alpha_{c}+\alpha_{a}=1
$$

where $k_{0}$ is the electrochemical rate constant [13,14], $\alpha_{c}$ and $\alpha_{a}$ are the cathodic and anodic transfer coefficients, respectively, $F, R$, and $T$ feature their usual significance, and $E_{f}^{0}$ is the formal potential. No flux boundary conditions are set at $\mathrm{r}=\mathrm{r}_{\max }$ and $\mathrm{z}=\mathrm{z}_{\max }$.

\subsection{Computational methods}

All numerical results were achieved via bespoke software written in $C++$ which solves the theoretical model via a Finite Differences approach. Thorough software validation is generally paramount in the development of all electrochemical software [15] and comprehensive software tests were carried out: Limiting cases were compared with analytical results as discussed below in the Figure 2 and 3, the conservation of mass is monitored in all simulations, and extensive convergence checks were carried out with respect to the step width of the discretisation set in space and time.

\subsection{Dimensionless coordinates}

To facilitate the comparison of theoretical findings with experimental data, theoretical results are wherever possible reported in the dimensionless coordinates [16] defined in Table 1.

\subsection{Modelling the voltammetry of hydrazine}

Numerical models of the hydrazine reaction use the parameters listed in Table 2. The values of the diffusion coefficient, the electrochemical rate constant, and the anodic transfer coefficient are determined experimentally as detailed in Section 4, 'results and discussion'. Furthermore and as shown below, the oxidation of hydrazine is a four-electron process with the first electron transfer being rate determining. The reaction can hence be modelled computationally as a modified oneelectron processes in which the current is evaluated for a rate-limiting one-electron transfer and subsequently multiplied by a factor of four to account for the four electrons that are transferred. 


\begin{tabular}{|c|c|c|}
\hline Parameter & Symbol (dimensioned) & Value \\
\hline Diffusion coefficient & $D$ & $1.31 \cdot 10^{-9} \mathrm{~m}^{2} \mathrm{~s}^{-1}$ \\
\hline Electrochemical rate constant and formal potential & $k_{0} e^{-\frac{\alpha_{a} F E_{f}^{0}}{R T}}$ & $2.19 \cdot 10^{-8} \mathrm{~m} \mathrm{~s}^{-1}$ \\
\hline Anodic transfer coefficient & $\alpha_{a}$ & 0.27 \\
\hline Particle radius [9] & $r_{p}$ & $4.3 \cdot 10^{-7} \mathrm{~m}$ \\
\hline Electrode radius & $r_{e}$ & $1.5 \cdot 10^{-3} \mathrm{~m}$ \\
\hline
\end{tabular}

Table 2: [Table was amended and reference was removed.] Parameters used for the numerical modelling of the cyclic voltammograms for the hydrazine oxidation. The diffusion coefficient, the electrochemical rate constant, and the anodic transfer coefficient are determined experimentally in this study. Please see Section 4, 'results and discussion' for all details.

\section{Results and discussion}

The following results and discussion section first presents the theoretical findings that are obtained from the numerical simulations. Next theory and experiment are compared using the paradigm of the relatively simple fully-irreversible hydrazine oxidation before, finally, we turn to experimental results of the more complex reactions: hexaammine ruthenium (III) $\mathrm{Ru}\left(\mathrm{NH}_{3}\right)_{6}{ }^{3+}$ reduction, ferrocenemethanol $\mathrm{FcCH}_{2} \mathrm{OH}$ oxidation, and ferrocyanide $\mathrm{Fe}(\mathrm{CN})_{6}{ }^{4-}$ oxidation.

\subsection{Theoretical results}

This section first investigates the potentials at which oxidative peaks occur in the calculated cyclic voltammograms. For a bare macroelectrode, well-established analytical theory exists for the reversibleand the irreversible limit [12]. In the reversible limit, i.e. at slow scan rates and high electrochemical rate constants, the peak potential converges to a constant value of:

$$
\theta_{\text {rev }}=\frac{2.218}{2}=1.109
$$

if $A$ and $B$ feature equal diffusion coefficients. This value remains unchanged for different scan rates as long as the electrochemical rate constant and the scan rate are chosen sufficiently close to the reversible limit. In contrast, the peak potential converges to a scan-rate dependent value in the irreversible limit, that is at fast scan rates and small electrochemical rate constants:

$$
\theta_{\text {irrev }}=\frac{1}{\alpha_{a}}\left(\ln \left(\frac{K_{0}}{\sqrt{\alpha_{a} \sigma}}\right)-0.78\right)
$$

While theory is readily available for the geometry of the bare macroelectrode, in the model of an electrode modified with inactive spheres, the mass transport is however complicated by the altered diffusion across the porous layer formed by the spheres and no analytical solutions have yet been found. The following therefore compares and contrasts the theoretical findings that are expressed in the Equations (6) and (7), numerical results for a bare macroelectrode, and numerical results for modified electrodes.

Figure 2 depicts plots of the oxidative peak potentials as a function of the scan rate for four different electrochemical rate constants $K_{0}$. In the Figure see an excellent agreement between the numerical results for the bare electrode and the corresponding theoretical predictions in the reversible and the irreversible limits as well as a smooth transition between the two limits at intermediate scan rates. For the modified electrodes however, the peak positions feature a more complex dependency on the scan rate. While at fast and slow scan rates the values for the bare electrode are approached, 
we - possibly counterintuitively - find the peak potentials elevated or reduced at intermediate scan rates and in comparison with the bare electrode. If the peak potential was falsely used as an indicator of the electrocatalytic activity of the spheres, one might wrongly infer positive or negative catalysis from the peak shifts though the electrode kinetics have remained entirely unchanged. This finding can be understood along the lines of our prior analysis [7] of a reversible reaction to which we refer the interested reader for full details beyond the following summary. In essence, four different diffusion mechanisms can be distinguished: (1) At slow scan rates, a thermal equilibrium is established across the porous layer formed by the spheres and the diffusion field resembles the diffusion field found at a bare electrode. (2) If the scan rate increases, the potential changes faster than the time that is required to reach the thermal equilibrium throughout the porous layer. The mass transport of analyte towards the electrode is then retarded by the presence of the spheres and the current peaks later than in the case of a bare electrode. As the time required to reach the thermal equilibrium across all layers increases with the number of layers, the elevated peak potentials for three layers of spheres are observed at lower scan rates than for one layer. (3) If the scan rate increases further, a thermal equilibrium is established only in the vicinity of the electrode surface while the spheres obstruct the mass transport of the analyte to the electrode on the time scale at which the thermal equilibrium is reached. The system then approaches the behaviour of a classic thin layer cell and the peak potential moves towards the formal potential. (4) In the limit of high scan rates, the diffusion field does not extend sufficiently far into the porous layer to affect the voltammetry and the peak potentials approach the values observed at a bare electrode.

Having discussed the potential at which the oxidative peak occurs, the following analysis turns towards the peak flux. As for the peak potentials, analytical theory has long been established for the geometry of a bare macroelectrode [12]. Randles-Ševčík equation for reversible reactions provides an analytical result for the peak flux as a function of the scan rate in the limit of a fast electrochemical rate constant and a slow scan rate:

$$
J_{\text {rev }}=0.446 \pi R_{\max }^{2} \sqrt{\sigma}
$$

while the irreversible Randles-Ševčík equation describes the peak flux in the limit of a fast scan rate and a low electrochemical rate constant:

$$
J_{\text {irrev }}=0.496 \pi R_{\max }^{2} \sqrt{\alpha_{a} \sigma}
$$

where we note that both expressions are functions of the scan rate featuring a $\sqrt{\sigma}$ proportionality. In analogy to the peak potentials, no analytical theory exists for the geometry of the modified electrodes and the following analysis must hence compare analytical theory in the limiting cases of a bare macroelectrode, numerical results for the bare macroelectrode, and numerical results for modified electrodes.

Figure 3 depicts the peak fluxes for different electrochemical rate constants as a function of the scan rate. As with the peak potentials, we find an excellent agreement of the numerical results for the bare electrode and the analytical values in the corresponding limiting cases: For small electrochemical rate constants and fast scan rates peak currents converge to the theoretical values for an irreversible system while for large electrochemical rate constants and slow scan rates we observe convergence to the results for the reversible case. At intermediate scan rates a smooth transition is found between the two limits. Significant differences can though be seen between the data for the bare and the modified electrodes. While, for slow and fast scan rates, the peak currents at the modified electrodes approach the peak currents seen at the bare electrodes, a notable drop in the peak flux is observed at intermediate scan rates. 

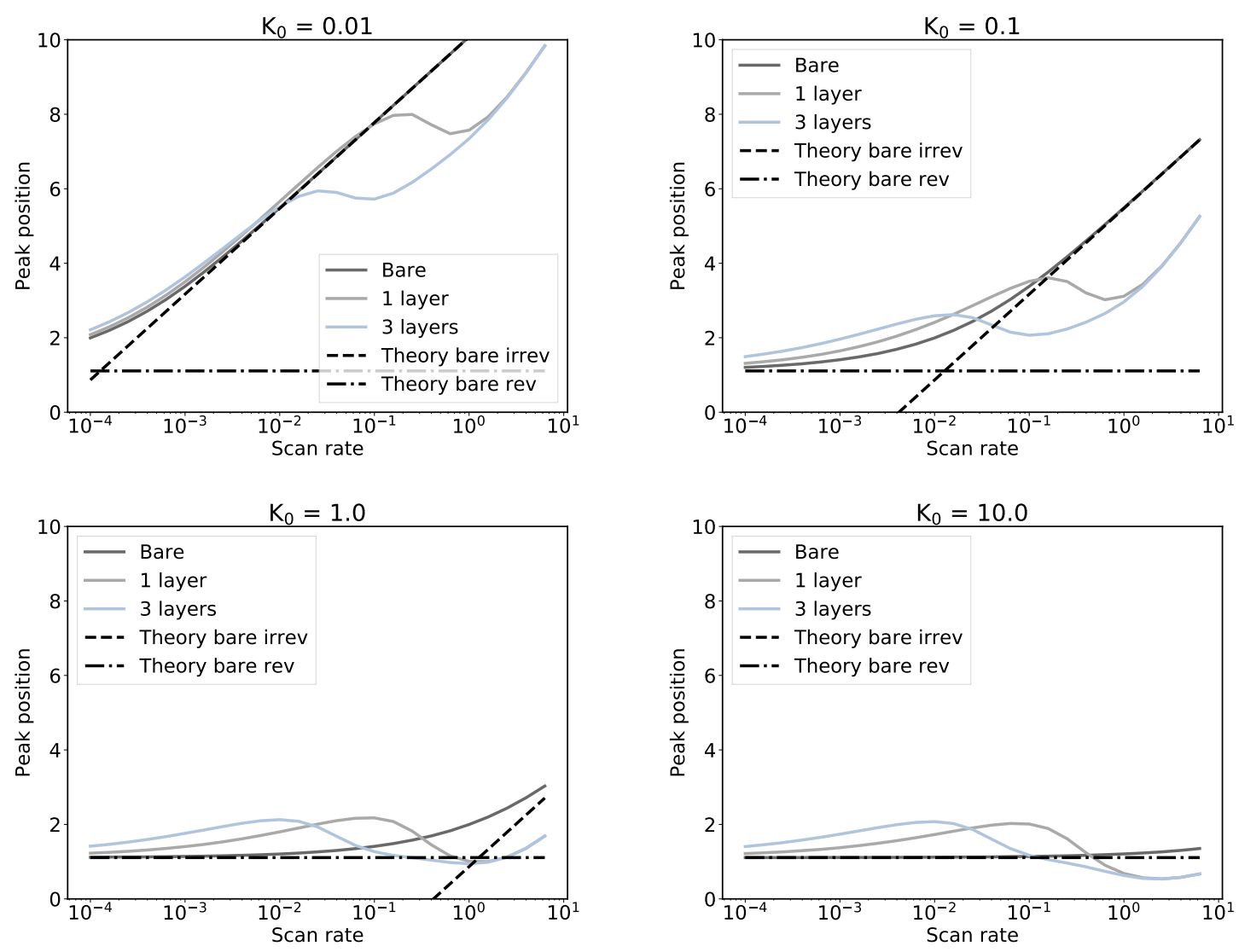

Figure 2: Oxidative peak potentials as a function of the scan rate evaluated for different dimensionless electrochemical rate constants $K_{0}$. The transfer coefficient $\alpha_{a}$ is set to 0.5 in the calculation of all depicted simulations.

The complex dependency of the peak flux on the scan rate can again be understood in analogy with the reversible case investigated in our previous work [7] to which we refer the interested reader. As discussed above and in respect to the peak positions, at slow and fast scan rates the voltammetry of the modified electrode converges to the voltammetry of the bare electrode: At slow scan rates a thermal equilibrium is established across the porous layer while, at fast scan rates, the diffusion field of the electrode does not extend sufficiently far into the porous layer for the spheres to have an impact on the voltammetry. In contrast and at intermediate scan rates, the mass transport of the analyte towards the electrode is obstructed and we find the peak currents reduced. This effect is more pronounced the more layers of spheres are deposited on the electrode.

\subsection{Validation of the theoretical results}

To validate our theoretical findings, we experimentally investigate the electrochemically fully irreversible oxidation of hydrazine at glassy carbon electrodes. The electrodes are herein modified with layers of Nafion particles [8-11] which, in a prior study [8], have been shown to be electrochemically inactive in aqueous solution. The oxidation of hydrazine in aqueous solution has been well-characterised in literature [17-21] and involves a four electron-transfer of which the first is the rate-determining step: 

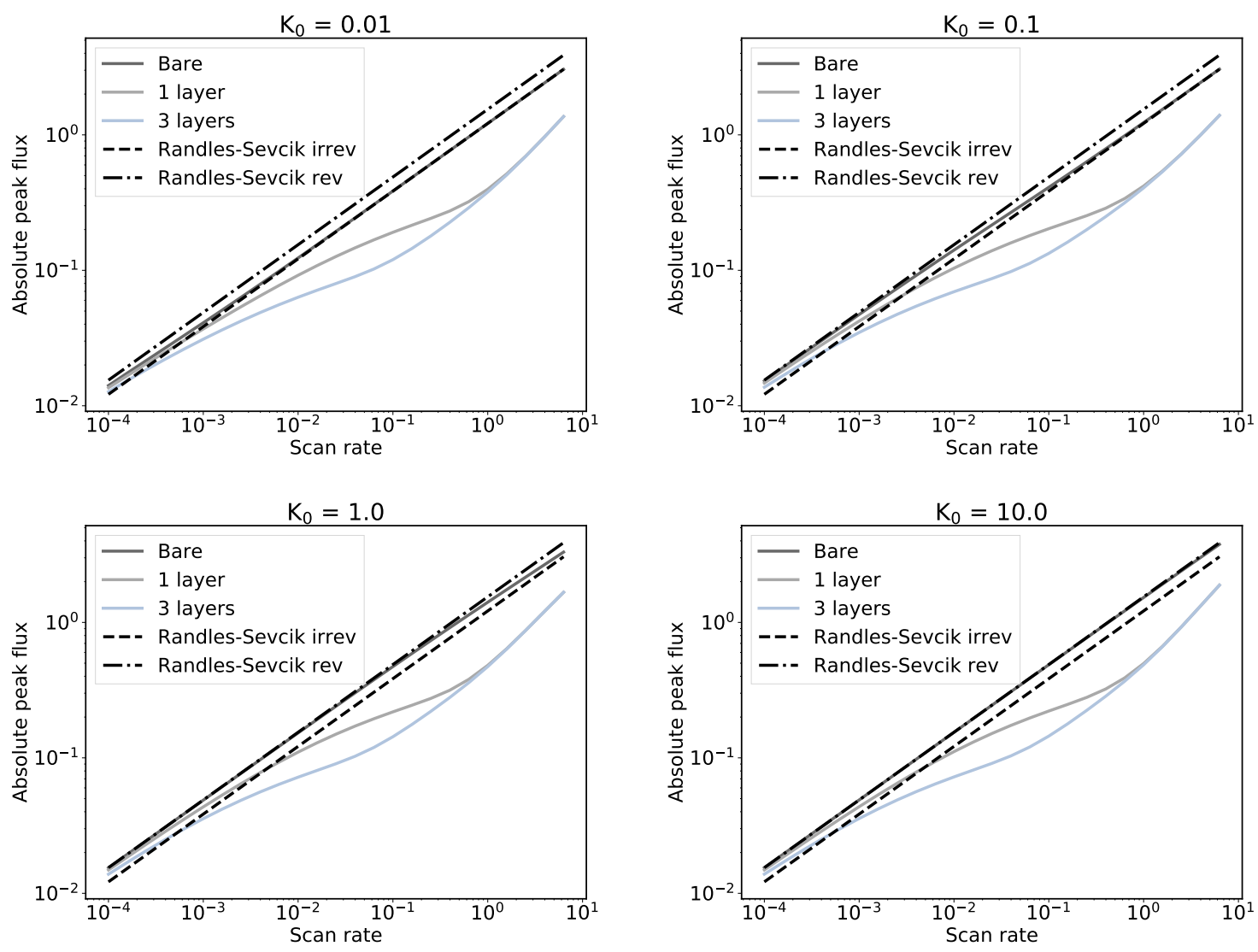

Figure 3: Oxidative peak fluxes as a function of the scan rate. Data were calculated for different electrochemical rate constants and a transfer coefficient $\alpha_{a}$ of 0.5 .

$$
\mathrm{N}_{2} \mathrm{H}_{5}^{+}+5 \mathrm{OH}^{-} \rightarrow \mathrm{N}_{2}+5 \mathrm{H}_{2} \mathrm{O}+4 e^{-}
$$

We note that the pKa of the protonated hydrazine, $\mathrm{N}_{2} \mathrm{H}_{5}{ }^{+}$, is 8.1 [22] and hydrazine is hence present in its protonated form in phosphate buffered saline solution at $\mathrm{pH}$ 7.4.

Voltammograms of the hydrazine oxidation are recorded at a bare electrode and at electrodes modified with Nafion particles for a wide range of scan rates. The voltammetry of the bare electrodes is subsequently analysed and compared with the theoretically predicted peak potentials and peak currents. Good agreement is seen as shown in the supporting information and the validity of the theoretical model of the electrode kinetics is hence proven. Via a Tafel analysis [23], the electrochemical transfer coefficient $\alpha_{a}[13,14]$ for hydrazine oxidation is determined to be $0.27 \pm 0.003$. On the basis of the multiple-electron-transfer irreversible Randles-Ševč́́k equation [12] the diffusion coefficient is determined to be $(1.31 \pm 0.03) \cdot 10^{-9} \mathrm{~m}^{2} \mathrm{~s}^{-1}$, which is in excellent agreement with prior literature $[17,19,24]$. The electrochemical rate constant $\mathrm{k}_{0}$ and the formal potential $E_{f}^{0}$ of the hydrazine oxidation are determined as a composite parameter as defined in Table 2. [text and reference were removed.]

Moving on to modified electrodes, Figure S5 compares voltammetry recorded at a bare electrode and electrode with an average coverage of one, three and five layers of Nafion particles. A strong dependency of the voltammograms on the amount of particles deposited is seen: There is a clear shift 

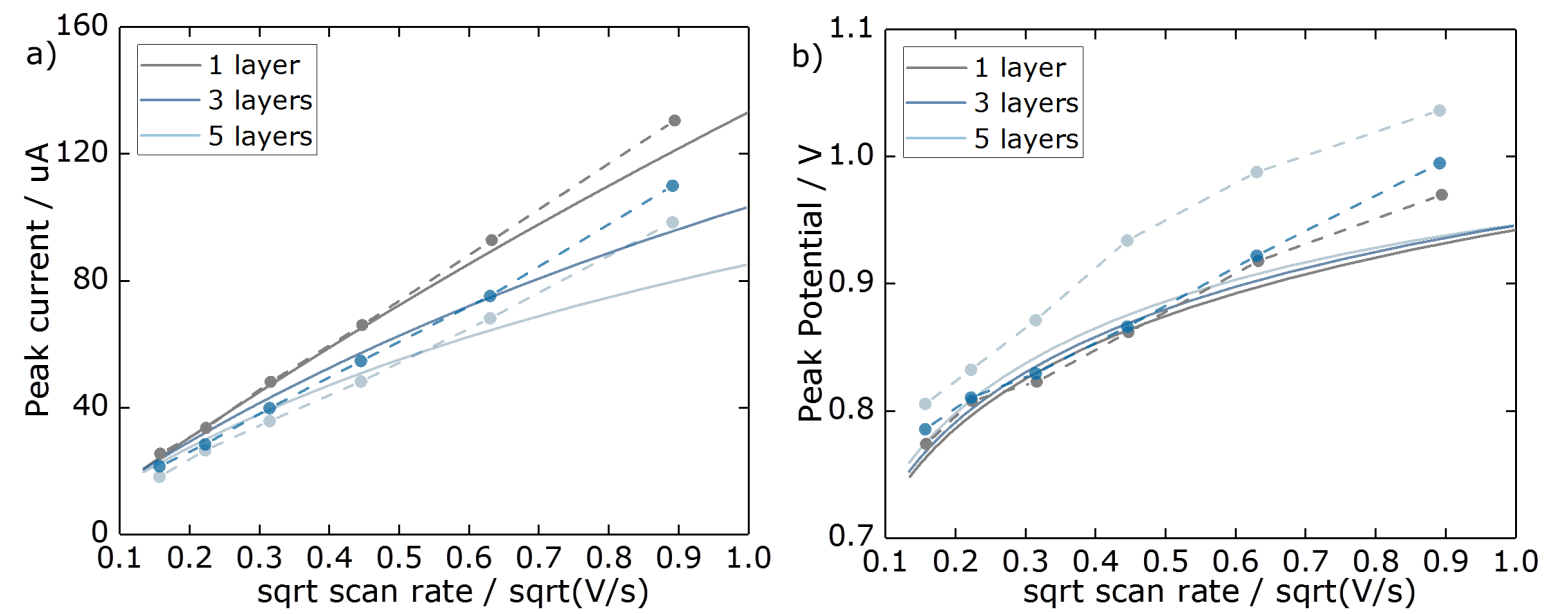

Figure 4: (a) Comparison of measured (dashed lines) and simulated (solid lines) peak currents as a function of scan rate for an average surface coverage of one, three and five layers of Nafion particles deposited on the electrode. (b) Comparison of measured (dashed lines) and simulated (solid lines) peak potentials.

in peak potential of hydrazine oxidation and a marked change in the peak current. Figure 4a shows the measured peak currents (dashed lines) plotted as a function of the scan rate and in comparison with simulated data (solid lines). In agreement with the theoretical prediction, we find significant variations with the scan rate and the number of layers deposited. The simulated trends are reproduced in the experimental data and, for one layer of particles, an almost perfect match between experiment and theory is observed. Differences are likely due to the assumptions made in the theoretical model - particularly the diffusion domain approximation and the assumption of perfect hexagonal packing and imperfections in the experiment: There is an inhomogeneity in the size and shape of synthesised Nafion particles, there are imperfections in the deposited layers, and there is potentially a limited electrolytic buffer support inside the porous layer.

Having shown the applicability of the theoretical model in terms of peak currents, we next investigate the peak potentials as a function of the scan rate for an average surface coverage of one, three, and five layers of Nafion particles as depicted in Figure 4b. The experimental data reproduces the trends seen in the simulations and validates the theoretical model: Following the theoretical prediction, significant variations are seen with the scan rate and at varied numbers of particle layers. The lowered peak currents and the elevated peak potential may intuitively and might, without modelling, falsely suggest a negative electrocatalytic activity of Nafion particles.

\subsection{More complex reactions}

In the following we discuss three different reactions all of which are more complex than the reaction of hydrazine as reactants or products adsorb onto the surfaces of the spheres or the reactant electrostatically interacts with the spheres. In contrast to the hydrazine oxidation which is fully electrochemically irreversible, all three systems are reversible.

\section{Hexaammineruthenium (III) reduction}

The above experimental data and our previous work [8] on reversible systems have confirmed the formerly theoretically-predicted complexity of any voltammetric analysis at nano-structured interfaces. 
To obtain insights into the catalytic properties of an electrode material, the voltammetric analysis of any particle-modified electrode crucially requires complementary theoretical modelling or data obtained via other experimental techniques. To further emphasise this fact, the following presents a more complex system that features electrostatic interactions. Nafion particles are negatively charged due to the many $\mathrm{SO}_{3}^{-}$groups of Nafion $[25,26]$. In contrast to the hydrazine oxidation where both the reactant (hydrazine) and the product (nitrogen) are charge neutral, hexaammineruthenium (III) is positively charged and hence likely affected by an attractive electrostatic interaction with the Nafion particles.

The formerly well-characterised [27] one-electron reduction of $\mathrm{Ru}\left(\mathrm{NH}_{3}\right)_{6}{ }^{3+}$ is studied at a bare glassy carbon electrode in an argon-saturated $0.1 \mathrm{M} \mathrm{KCl}$ solution containing $1.0 \mathrm{mM}\left[\mathrm{Ru}\left(\mathrm{NH}_{3}\right)_{6}\right] \mathrm{Cl}_{3}$, see supporting information. Via the reversible Randles-Ševčík equation, the diffusion coefficient is found to be $(8.34 \pm 0.14) \cdot 10^{-10} \mathrm{~m}^{2} \mathrm{~s}^{-1}$ which matches priorly reported value [28] of $((8.43 \pm 0.03)$ $\cdot 10^{-10} \mathrm{~m}^{2} \mathrm{~s}^{-1}$. The formal potential is estimated to be $-0.176 \mathrm{~V}$ vs SCE via the determination of the mid-point potential.

Figure 5 compares the voltammetry of a bare glassy carbon electrode and modified electrodes with one layer and three layers of Nafion particles. As one would anticipate from the analysis of the voltammetry of hydrazine and our previous work [8], a strong dependency of the voltammograms on the amount of Nafion particles deposited and scan rate is observed. Most notably, there is shift in the position of forward peak towards higher overpotentials and a dramatic increase in the peak current. This is likely due to the attractant interaction between $\mathrm{Ru}\left(\mathrm{NH}_{3}\right)_{6}{ }^{3+}$ molecules and Nafion particles leading to the adsorption of $\mathrm{Ru}\left(\mathrm{NH}_{3}\right)_{6}{ }^{3+}$ onto the Nafion particle surface and probably into the bulk of the particles [11]. Therefore, as the amount of Nafion particles deposited on electrode surface increases, the number of adsorbed reactant molecules increases which results in the peak appearing at higher overpotentials and larger peak currents. The higher overpotentials are herein likely due to the retarded mass transport inside the porous layer and the slow release of the adsorbed reactant while the elevated peak current may result from the net preconcentration of the reactant inside the porous layer. In terms of the reverse scan the peak potential shifts to lower overpotentials whilst a higher peak current is seen. The increase in the peak current likely relates to the increased amount of $\mathrm{Ru}\left(\mathrm{NH}_{3}\right)_{6}{ }^{2+}$ formed on the forward scan. During the reverse scan, the $\mathrm{Ru}\left(\mathrm{NH}_{3}\right)_{6}{ }^{2+}$ undergoes an oxidation to form the more positive-charged $\mathrm{Ru}\left(\mathrm{NH}_{3}\right)_{6}{ }^{3+}$ which is affected by a stronger attractive interaction with Nafion particles. This may lead to this process being apparently more favoured primarily thermodynamically due to the $\mathrm{Ru}$ (II/III) couple interacting with the $\mathrm{SO}_{3}{ }^{-}$- and causing the lower overpotentials observed.

\section{Ferrocenemethanol oxidation}

In the following, we investigate another relatively simple and well-studied reaction: The oxidation of ferrocenemethanol at glassy carbon electrodes. The electrodes are again modified with Nafion particles which induces different electrostatic interactions: In this system, the reactant is charge neutral while the product is positively charged and hence features an attractive interaction with the Nafion particles.

In analogy with prior sections, the oxidation of ferrocenemethanol is first examined at a bare electrode as discussed in the supporting information. The diffusion coefficient is found to be $(7.61 \pm$ $0.18) \cdot 10^{-10} \mathrm{~m}^{2} \mathrm{~s}^{-1}$, in excellent agreement with prior literature value [29] of $(7.6 \pm 0.4) \cdot 10^{-10} \mathrm{~m}^{2}$ $\mathrm{s}^{-1}$, and the formal potential is found to be $0.19 \mathrm{~V}$ vs SCE.

Voltammograms are recorded at different scan rates at electrodes modified with one, two, and three layers of Nafion particles. The measured peak currents and peak potentials are plotted as a 

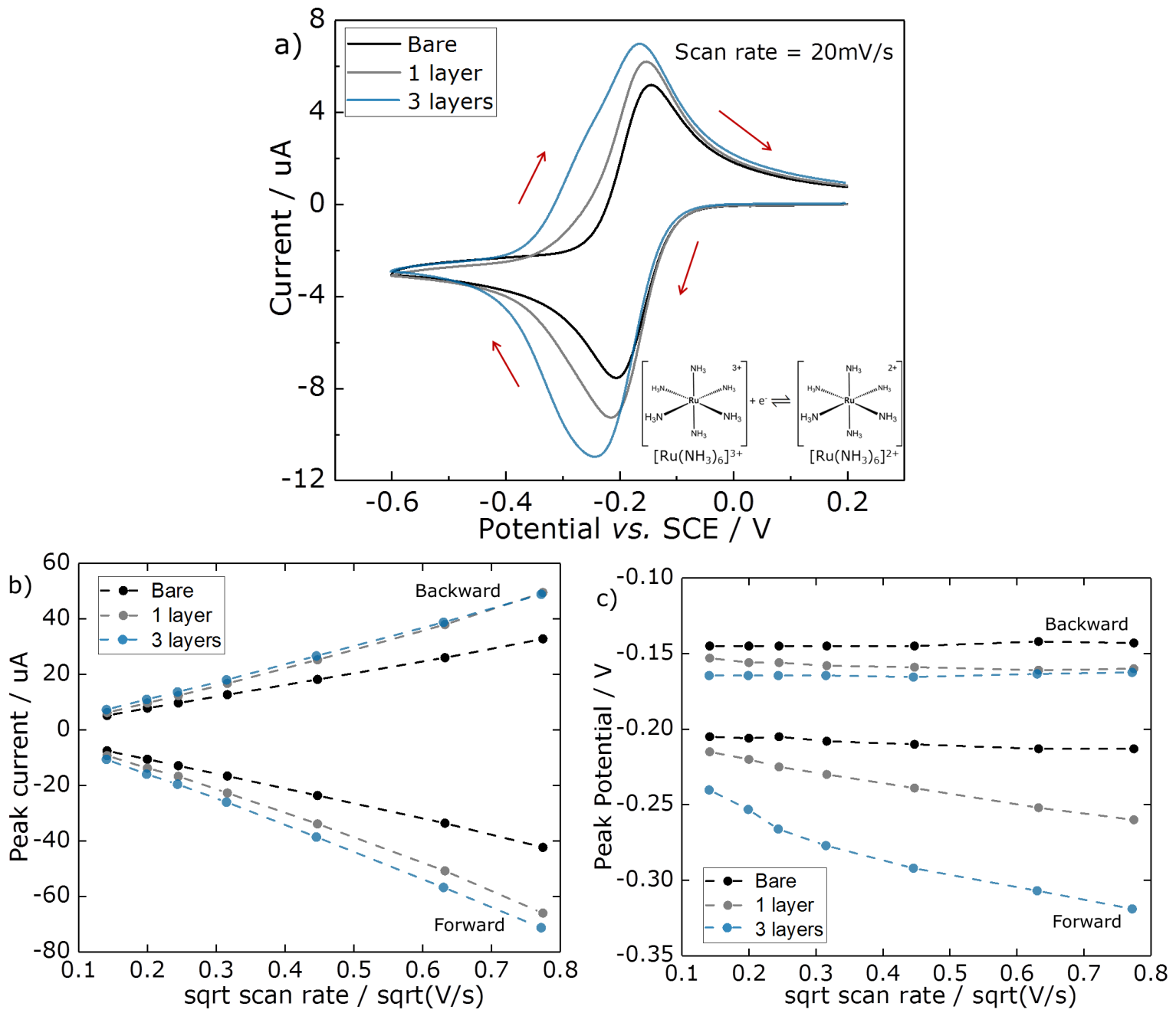

Figure 5: (a) Representative voltammograms at a bare glassy carbon macroelectrode and modified electrodes in $0.1 \mathrm{M} \mathrm{KCl}$ containing $1 \mathrm{mM}\left[\mathrm{Ru}\left(\mathrm{NH}_{3}\right)_{6}\right] \mathrm{Cl}_{3}$ recorded at $20 \mathrm{mV} \mathrm{s}^{-1}$. The scan begins at a potential of $200 \mathrm{mV}$ and reverses at a potential of $-600 \mathrm{mV}$. (b) Comparison of measured (dashed lines) and simulated (solid lines) peak currents as a function of scan rate for an average surface coverage of one and three layers of Nafion particles deposited on the electrode. (b) Comparison of measured (dashed lines) and simulated (solid lines) peak potentials.

function of the scan rate and compared with the bare electrode data as depicted in Figure 6. Here, a different dependency of the voltammograms on the amount of Nafion particles deposited is observed: There is a shift in the position of forward peak towards lower overpotentials and a decrease in the peak current. As the reactant is not charged and does not interact electrostatically with the Nafion spheres, the reduced peak currents are attributed to the retarded mass transport of the analyte towards the electrode in analogy with the hydrazine results. The oxidation product $\mathrm{FcCH}_{2} \mathrm{OH}^{+}$is positively charged and affected by attractive electrostatic interactions with the Nafion spheres. This results in the oxidation process which is electrochemically reversible being more favoured and hence the peak potentials being reduced. Moreover, this attractive interaction between the oxidation product and the spheres leads to the adsorption and accumulation of the $\mathrm{FcCH}_{2} \mathrm{OH}^{+}$onto the Nafion surface and/or bulk. In the reverse scans, the complex dependency of the peak currents and peak potential 

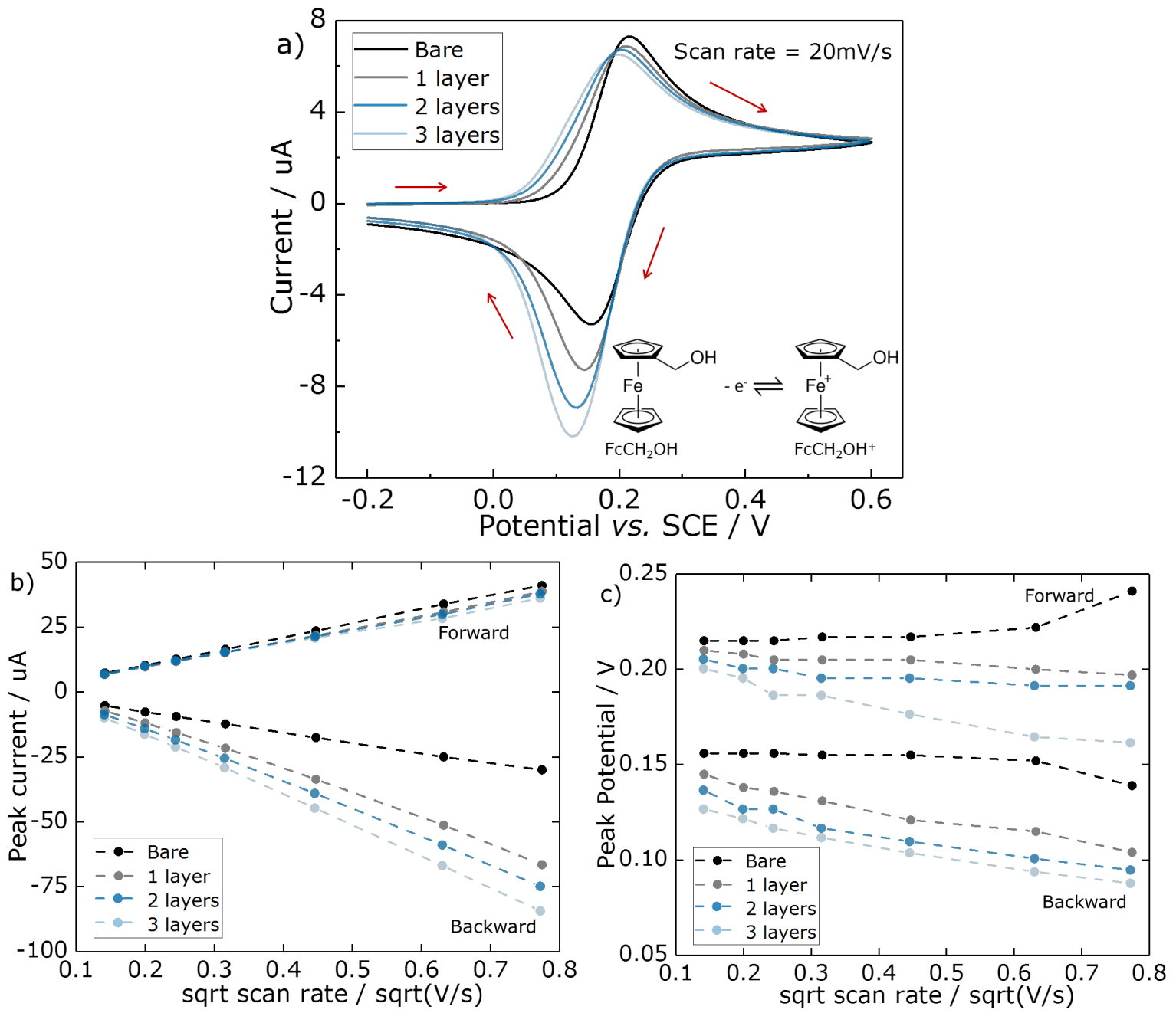

Figure 6: (a) Representative voltammograms of a bare glassy carbon macroelectrode or Nafion particlesmodified electrodes in $0.1 \mathrm{M} \mathrm{KCl}$ containing $1 \mathrm{mM}$ ferrocenemethanol recorded at $20 \mathrm{mV} \mathrm{s}^{-1}$. The scan begins at a potential of $-200 \mathrm{mV}$ and reverses at a potential of $600 \mathrm{mV}$. (b) Comparison of measured (dashed lines) and simulated (solid lines) peak currents as a function of scan rate for an average surface coverage of one, two and three layers of Nafion particles deposited on the electrode. (b) Comparison of measured (dashed lines) and simulated (solid lines) peak potentials.

on the amount of Nafion sphere deposited can therefore be understood in analogy with the reduction of $\mathrm{Ru}\left(\mathrm{NH}_{3}\right)_{6}{ }^{3+}$.

\section{Ferrocyanide oxidation}

Having shown the complexity of an attractive interaction between the reactant or the product and the spheres, the following investigates the reaction of ferrocyanide where there are repulsive electrostatic forces between the reactant and the spheres.

To this end, we first study the oxidation of ferrocyanide $\left[\mathrm{Fe}(\mathrm{CN})_{6}\right]^{4-}$ at a bare glassy carbon electrode as shown in the supporting information. The diffusion coefficient is found to be $(6.27 \pm$ $0.17) \cdot 10^{-10} \mathrm{~m}^{2} \mathrm{~s}^{-1}$ which is in good agreement with the literature value [30] of $(6.6 \pm 0.3) \cdot 10^{-10}$ $\mathrm{m}^{2} \mathrm{~s}^{-1}$ while the formal potential is found to be $0.182 \mathrm{~V}$ vs SCE. 


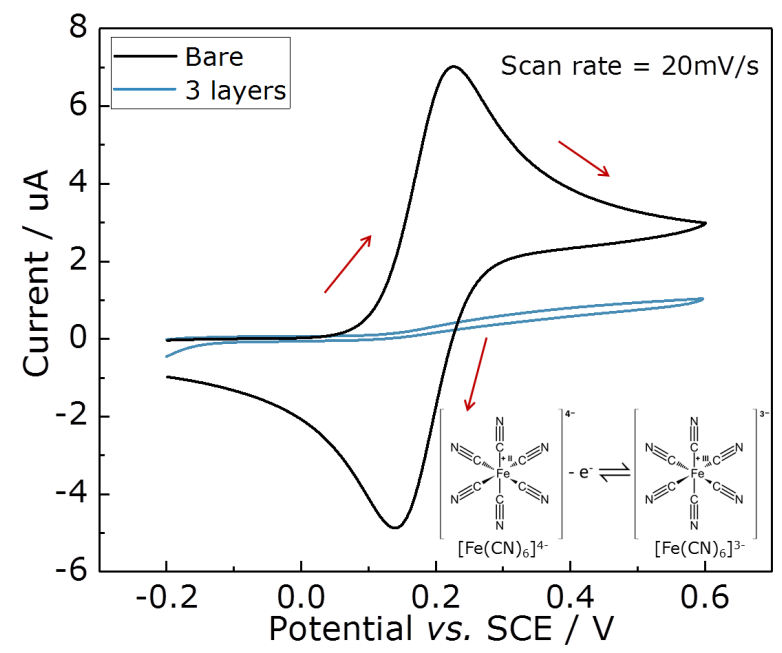

Figure 7: Representative voltammograms of a bare glassy carbon macroelectrode or the electrodes modified with three layers of Nafion particles in $0.1 \mathrm{M} \mathrm{KCl}$ containing $1 \mathrm{mM}$ ferrocyanide recorded at $20 \mathrm{mV}$ $\mathrm{s}^{-1}$. The scan begins at a potential of $-200 \mathrm{mV}$ and reverses at a potential of $600 \mathrm{mV}$.

Figure 7 compares the voltammetry of the ferrocyanide oxidation at a bare electrode and at an electrode modified with three layers of Nafion particles. The latter shows a dramatic reduction in current and no clear peak can be seen, which is attributed to powerful electrostatic repulsion between the charged reactant and the Nafion leading to the reaction being almost fully blocked.

\section{Conclusions}

This work has shown that the voltammetry of particle-modified electrodes bears a significant level of complexity. In cyclic voltammetry, altered mass transport inside the porous layers formed by the nanoparticles may lead to shifts in peak potentials and peak currents which may falsely be interpreted as a positive or negative catalytic activity of the deposited particles. While prior studies focussed on electrochemically-reversible reactions, the first part of this study investigates quasi- and irreversible reactions and reveal the complex dependency of voltammetric features on the scan rate and the amount of particles deposited. Our results demonstrate that any voltammetric analysis of such data crucially requires complementary theoretical modelling to extract any meaningful information from experimental data.

In addition and in the second part of this work, we have experimentally demonstrated the impact of electrostatic effects on the voltammetry of three reversible systems: If reactants or products and the particle surfaces are charged, entirely different voltammetric features are observed which again shows the profound influence on the voltammetry exerted by modifying particles and signalling care is needed in any interpretation.

\section{Data Availability}

The raw/processed data required to reproduce these findings cannot be shared at this time due to technical or time limitations. 


\section{References}

[1] L. Liu and A. Corma. Metal Catalysts for Heterogeneous Catalysis: From Single Atoms to Nanoclusters and Nanoparticles. Chemical Reviews, 118(10):4981-5079, 2018.

[2] L. Zhang, Z.-J. Zhao, and J. Gong. Nanostructured materials for heterogeneous electrocatalytic $\mathrm{CO}_{2}$ reduction and their related reaction mechanisms. Angewandte Chemie International Edition, 56(38):11326-11353, 2017.

[3] F. Wang, X. Wu, C. Li, Y. Zhu, L. Fu, Y. Wu, and X. Liu. Nanostructured positive electrode materials for post-lithium ion batteries. Energy \& Environmental Science, 9(12):3570-3611, 2016.

[4] Q. Lu, J. G. Chen, and J. Q. Xiao. Nanostructured electrodes for high-performance pseudocapacitors. Angewandte Chemie International Edition, 52(7):1882-1889, 2013.

[5] Y. Li and G. A. Somorjai. Nanoscale advances in catalysis and energy applications. Nano Letters, 10(7):2289-2295, 2010.

[6] R. W. Murray. Nanoelectrochemistry: Metal nanoparticles, nanoelectrodes, and nanopores. Chemical Reviews, 108(7):2688-2720, 2008.

[7] E. Kätelhön, L. Chen, and R. G. Compton. Nanoparticle electrocatalysis: Unscrambling illusory inhibition and catalysis. Applied Materials Today, 15:139-144, 2019.

[8] L. Chen, E. Kätelhön, and R. G. Compton. Unscrambling illusory inhibition and catalysis in nanoparticle electrochemistry: Experiment and theory. Applied Materials Today, 16:141-145, 2019.

[9] H. Yang, X. Li, C. Batchelor-McAuley, S. V. Sokolov, and R. G. Compton. Nafion particles doped with methyl viologen: Electrochemistry. Physical Chemistry Chemical Physics, 20(1):682-689, 2017.

[10] L. Chen, C. Lin, and R. G. Compton. Single entity electrocatalysis: Oxygen reduction mediated via methyl viologen doped Nafion nanoparticles. Physical Chemistry Chemical Physics, 20(23):15795-15806, 2018.

[11] L. Chen, C. Lin, and R. G. Compton. Electrochemical characterisation and comparison of transport in Nafion films and particles. Physical Chemistry Chemical Physics, 21(2):607-616, 2019.

[12] R. G. Compton and C. E. Banks. Understanding voltammetry (third edition). World Scientific Publishing, 2018.

[13] R. Guidelli, R. G. Compton, J. M. Feliu, E. Gileadi, J. Lipkowski, W. Schmickler, and S. Trasatti. Defining the transfer coefficient in electrochemistry: An assessment (IUPAC technical report). Pure and Applied Chemistry, 86(2):245-258, 2014.

[14] R. Guidelli, R. G. Compton, J. M. Feliu, E. Gileadi, J. Lipkowski, W. Schmickler, and S. Trasatti. Definition of the transfer coefficient in electrochemistry (IUPAC recommendations 2014). Pure and Applied Chemistry, 86(2):259-262, 2014.

[15] E. Kätelhön and R. G. Compton. Testing and validating electroanalytical simulations. Analyst, 140(8):2592-2598, 2015. 
[16] R. G. Compton, E. Laborda, and K. R. Ward. Understanding voltammetry: Simulation of electrode processes. Imperial College Press, 2014.

[17] S. Karp and L. Meites. The Voltammetric Characteristics and Mechanism of Electroöxidation of Hydrazine. American Chemical Society, 84(6):906-912, 1962.

[18] Y.-D. Zhao, W.-D. Zhang, H. Chen, and Q.-M. Luo. Anodic oxidation of hydrazine at carbon nanotube powder microelectrode and its detection. Talanta, 58(3):529-534, 2002.

[19] S. M. Golabi and H. R. Zare. Electrocatalytic oxidation of hydrazine at a chlorogenic acid (CGA) modified glassy carbon electrode. Journal of Electroanalytical Chemistry, 465(2):168-176, 1999.

[20] H. Xia and H.-L. Li. Electrooxidation of hydrazine catalyzed by 4-hydroxy-2,2,6,6-tetramethylpiperidinyloxy (TEMPOL). Journal of Electroanalytical Chemistry, 430(1):183-187, 1997.

[21] H. R. Zare and A. M. Habibirad. Electrochemistry and electrocatalytic activity of catechin film on a glassy carbon electrode toward the oxidation of hydrazine. Journal of Solid State Electrochemistry, 10(6):348-359, 2006.

[22] H. K. Hall. Correlation of the base strengths of amines ${ }^{1}$. Journal of the American Chemical Society, 79(20):5441-5444, 1957.

[23] D. Li, C. Lin, C. Batchelor-McAuley, L. Chen, and R. G. Compton. Tafel analysis in practice. Journal of Electroanalytical Chemistry, 826:117-124, 2018.

[24] U. Scharf and E. W. Grabner. Electrocatalytic oxidation of hydrazine at a Prussian Blue-modified glassy carbon electrode. Electrochimica Acta, 41(2):233-239, 1996.

[25] A. Kusoglu and A. Z. Weber. New insights into perfluorinated sulfonic-acid ionomers. Chemical Reviews, 117(3):987-1104, 2017.

[26] K. A. Mauritz and R. B. Moore. State of understanding of nafion. Chemical Reviews, 104(10):4535-4586, 2004.

[27] L. Chen and R. G. Compton. Reference electrodes for electrochemical sensors based on redox couples immobilized within nafion films. ACS Sensors, 4(6):1716-1723, 2019.

[28] Y. Wang, J. G. Limon-Petersen, and R. G. Compton. Measurement of the diffusion coefficients of $\left[\mathrm{Ru}\left(\mathrm{NH}_{3}\right)_{6}\right]^{3+}$ and $\left[\mathrm{Ru}\left(\mathrm{NH}_{3}\right)_{6}\right]^{2+}$ in aqueous solution using microelectrode double potential step chronoamperometry. Journal of Electroanalytical Chemistry, 652(1):13-17, 2011.

[29] C. Amatore, N. Da Mota, C. Sella, and L. Thouin. Theory and experiments of transport at channel microband electrodes under laminar flows. 1. steady-state regimes at a single electrode. Analytical Chemistry, 79(22):8502-8510, 2007.

[30] S. J. Konopka and B. McDuffie. Diffusion coefficients of ferri- and ferrocyanide ions in aqueous media, using twin-electrode thin-layer electrochemistry. Analytical Chemistry, 42(14):1741-1746, 1970 . 